$11-1-2012$

\title{
Class(es) of Factor-Type Estimator(s) in Presence of Measurement Error
}

\author{
Diwakar Shukla \\ Dr. Hari Singh Gour University, Sagar, M. P., India \\ Sharad Pathak \\ Dr. Hari Singh Gour University, Sagar, M. P., India \\ Narendra Singh Thakur \\ Banasthali University, Rajasthan, India
}

Follow this and additional works at: http://digitalcommons.wayne.edu/jmasm

Part of the Applied Statistics Commons, Social and Behavioral Sciences Commons, and the Statistical Theory Commons

\section{Recommended Citation}

Shukla, Diwakar; Pathak, Sharad; and Thakur, Narendra Singh (2012) "Class(es) of Factor-Type Estimator(s) in Presence of Measurement Error," Journal of Modern Applied Statistical Methods: Vol. 11 : Iss. 2 , Article 6.

DOI: $10.22237 /$ jmasm/1351742700

Available at: http://digitalcommons.wayne.edu/jmasm/vol11/iss2/6

This Regular Article is brought to you for free and open access by the Open Access Journals at DigitalCommons@WayneState. It has been accepted for inclusion in Journal of Modern Applied Statistical Methods by an authorized editor of DigitalCommons@WayneState. 


\title{
Class(es) of Factor-Type Estimator(s) in Presence of Measurement Error
}

\author{
Diwakar Shukla Sharad Pathak \\ Narendra Singh Thakur \\ Dr. Hari Singh Gour University, \\ Sagar, M. P., India \\ Banasthali University, \\ Rajasthan, India
}

When data is collected via sample survey it is assumed whatever is reported by a respondent is correct. However, given the issues of prestige bias, personal respect and honor, respondents' self-reported data often produces over- or under- estimated values as opposed to true values regarding the variables under question. This causes measurement error to be present in sample values. This article considers the factortype estimator as an estimation tool and examines its performance under a measurement error model. Expressions of optimization are derived and theoretical results are supported by numerical examples.

Key words: Measurement error, factor-type estimator, bias, mean squared error.

Introduction

Sample surveys result in an efficiency of estimators on the basis of collected or simulated data. Data for analyses may originate from various sampling sources, such as, simple random sampling, stratified sampling, systematic sampling or cluster sampling. Estimation methods are typically analyzed under the assumption that observations collected are true and without error; however, real life data, gathered through sample surveys contains errors due to memory failure, prestige bias, over reporting patterns, unwillingness to respond, desire for secrecy and other reasons. The deviation between true and observed values is error and is technically termed measurement error. Measurement error may be characterized as the difference between the value of a variable provided by the respondent and the true value of the same variable. The total survey error of a statistic with measurement error has both fixed error (bias) and variable error (variance) over repeated trials of the survey (Cochran, 2005;

Diwakar Shukla is an Associate Professor in the Department of Mathematics and Statistics. Email him at: diwakarshukla@rediffmail.com. Sharad Pathak is a Research Scholar in the Department of Mathematics and Statistics. Email him at: sharadpathakstats@yahoo.com. Narendra S. Thakur is an Assistant Professor at Banasthali University, Rajasthan.
Sukhatme, et al., 1984). Figure 1 illustrates the concept of measurement error.

There are two possibilities for incompleteness in a survey: incorrect response or non-response. Measurement bias provides a systematic pattern in the difference between the respondent's answers to a question and the correct answer. For example, a respondent may forget to report a few specific income sources resulting in total reported income being lower than actual. Measurement variance reflects random variation in answers provided to an interviewer while asking the same question, that is, often the same respondent provides different answers to the same question when asked repeatedly. Several methods are available in the survey sampling literature to handle nonresponse, including the revisit method, imputation methods, auxiliary sources utilization method and the neighboring units manipulation methods, however, when a respondent provides incorrect information regarding a variable, additional techniques are required. This study considers this aspect and deals with mean estimation under measurement error.

Manisha and Singh (2001) examined population mean estimation in the presence of measurement errors; they provided an effect of measurement errors on a new estimator obtained as a combination of ratio and mean per unit estimator. Shalabh (1997) studied a ratio method of estimation in the presence of measurement errors. Singh and Shukla (1987) presented a 


\section{DIWAKAR SHUKLA, SHARAD PATHAK \& NARENDRA SINGH THAKUR}

family of factor-type ratio estimators. Shukla (2002) proposed a new strategy for estimation in the form of a factor-type ratio estimator in two phase sampling. Shukla, et al. (2009) also proposed a mean estimation under imputation of missing data using factor-type estimator in twophase sampling and have since suggested a linear combination based imputation method for missing data in sample (Shukla, et al., 2011). Shukla, et al. (2012A) proposed an estimation of population mean using two auxiliary sources in sample surveys. Shukla, et al. (2012B) suggested an estimator for mean estimation in the presence measurement error of observations. Shukla, et al. (2012C) presented a transformed estimator for estimation of population mean with missing data in sample-surveys. Thakur, et al. (2011, 2012) suggested imputation strategies under double sampling.

Figure 1: Concept of Measurement Error

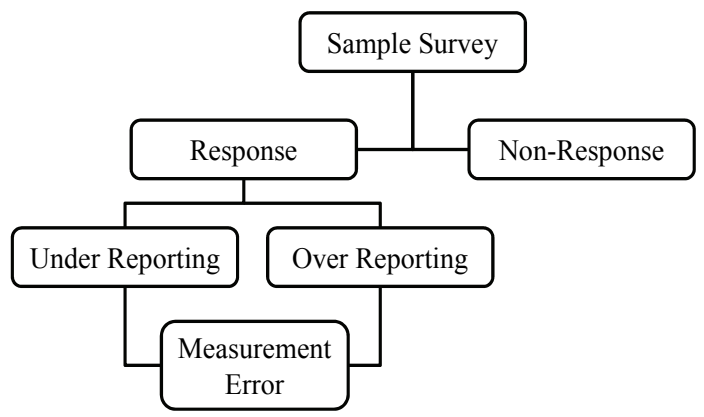

Singh and Karpe (2008a) presented a ratio-product estimator for population mean in the presence of measurement errors, Neter (1970) examined measurement errors in reports of consumer expenditures, Sud and Shrivastava (2000) studied estimation and population mean in repeat surveys on the presence of measurement errors and Sud, et al. (2001) considered a biased estimator in repeat surveys. Dalabehara and Sahoo (2000) and Kadilar and Cingi (2005) suggested estimators using two auxiliary sources in survey sampling. Other useful contributions over applications of measurement error models are provided by Fuller (1987), Cochran (1993), Mukhopadhyay
(2000), Murthy (1977), Sukhatme et al. (1984) and Cheng and Van Ness (1999). This article presents an estimation strategy under a measurement error model using two auxiliary sources for the purpose of optimization.

Study Notations and Assumptions

Assume a set of information obtained via a simple random sampling procedure on three characteristics $Y, X_{1}$ and $X_{2}$. Suppose $\left(y_{i}, x_{1_{i}}, x_{2_{i}}\right)$ are observational values and $\left(Y_{i}, X_{1_{i}}, X_{2_{i}}\right)$ are corresponding true values for the characteristics respectively. Notations for this study are:

$\bar{Y}, \bar{X}_{1}$ and $\bar{X}_{2}:$ Population parameters;

$\bar{y}, \bar{x}_{1}$ and $\bar{x}_{2}$ : Mean per unit estimates for a simple random sample of size $n$;

$n$ : Sample size;

$f$ : Sampling friction $(f=n / N)$;

$N$ : Population size;

$U_{i}:$ Measurement error for $Y$;

$V_{i}:$ Measurement error for $X_{i}$;

$T_{i}$ : Measurement error for $X_{2}$;

$\sigma_{U}^{2}, \sigma_{V}^{2}$ and $\sigma_{T}^{2}$ : Variances for measurement error;

$\sigma_{Y}^{2}, \sigma_{X_{1}}^{2}$ and $\sigma_{X_{2}}^{2}:$ Variances of variable $Y, X_{I}$ and $X_{2}$ respectively;

$\rho_{01}$ : Correlation between variable $Y$ and $X_{I}$;

$\rho_{02}$ : Correlation between variable $Y$ and $X_{2}$;

$\rho_{12}$ : Correlation between variable $X_{1}$ and $X_{2}$; 
$C_{Y}=\sigma_{Y} / \bar{Y}:$ Coefficient of variation for variable $Y\left(C_{0}\right)$;

$C_{X_{1}}=\sigma_{X_{1}} / \bar{X}_{1}:$ Coefficient of variation for variable $X_{1}\left(C_{1}\right)$; and

$C_{X_{2}}=\sigma_{X_{2}} / \bar{X}_{2}:$ Coefficient of variation for variable $X_{2}\left(C_{2}\right)$.

New notations are:

$$
\begin{gathered}
W_{Y}=\frac{\sum\left(Y_{i}-\bar{Y}\right)}{\sqrt{n}}, \\
W_{X_{1}}=\frac{\sum\left(X_{1_{i}}-\bar{X}_{1}\right)}{\sqrt{n}}, \\
W_{X_{2}}=\frac{\sum\left(X_{2_{i}}-\bar{X}_{2}\right)}{\sqrt{n}}, \\
W_{U}=\frac{\sum\left(U_{i}\right)}{\sqrt{n}}, \\
W_{V}=\frac{\sum\left(V_{i}\right)}{\sqrt{n}},
\end{gathered}
$$

and

$$
W_{T}=\frac{\sum\left(T_{i}\right)}{\sqrt{n}} \text {. }
$$

Assume the measurement errors are stochastic in nature and are uncorrelated, the sum of measurement error is zero and the variances are $\sigma_{U}^{2}, \sigma_{V}^{2}$ and $\sigma_{T}^{2}$, respectively. For an $i^{\text {th }}$ unit $(i=1,2,3, \ldots, n)$ unit in the sample assume the measurement errors are:

$$
\left.\begin{array}{l}
U_{i}=y_{i}-Y_{i} \\
V_{i}=x_{1_{i}}-X_{1_{i}} \\
T_{i}=x_{2_{i}}-X_{2_{i}}
\end{array}\right\}
$$

and, from (3.1),

$$
\bar{y}_{i}=\frac{1}{n} \sum_{i}\left(U_{i}+Y_{i}\right)
$$

or

$$
\bar{y}-\bar{Y}=\frac{W_{Y}}{\sqrt{n}}+\frac{W_{U}}{\sqrt{n}} .
$$

Similarly,

$$
\bar{x}_{1}-\bar{X}_{1}=\frac{W_{X_{1}}}{\sqrt{n}}+\frac{W_{V}}{\sqrt{n}}
$$

and

$$
\bar{x}_{2}-\bar{X}_{2}=\frac{W_{X_{2}}}{\sqrt{n}}+\frac{W_{T}}{\sqrt{n}} .
$$

Existing Estimators: Mean per Unit Estimator

The mean per unit (or mean) estimator is a well-known estimator, and in the setup of measurement error, $\bar{y}_{i}=n^{-1} \sum_{i}\left(U_{i}+Y_{i}\right)$, is shown in (3.2). The bias for $\bar{y}$ is zero, that is,

$$
E(\bar{y})=E\left[\frac{1}{n} \sum_{i}\left(U_{i}+Y_{i}\right)\right]=(\bar{Y})
$$

and the variance is

$$
\operatorname{Variance}(\bar{y})=\frac{\sigma_{Y}^{2}}{n}\left[1+\frac{\sigma_{U}^{2}}{\sigma_{Y}^{2}}\right]
$$

To estimate $\bar{Y}$, the sample statistic $\bar{y}$, which provides an unbiased estimator, can be used. In mean per unit estimator $\bar{y}$ no additional information is required. Several methods exist for using the auxiliary $X$ characteristic. 
Existing Estimators: Shalabh (1997) Estimator

Shalabh (1997) proposed an estimator that is a ratio estimator studied under measurement error.

$$
t_{R}=\frac{\bar{y}}{\bar{x}} \cdot \mu_{X}
$$

Where the bias of $t_{R}$ is

$$
B\left(t_{R}\right)=\frac{\mu_{Y}}{n}\left[C_{X}\left(C_{X}-\rho C_{Y}\right)+\frac{\sigma_{v}^{2}}{\mu_{X}^{2}}\right]
$$

and the mean squared error is

$$
\begin{aligned}
& \operatorname{MSE}\left(t_{R}\right)= \\
& \frac{\sigma_{Y}^{2}}{n}\left[1-\frac{C_{X}}{C_{Y}}\left(2 \rho-\frac{C_{X}}{C_{Y}}\right)\right]+\frac{1}{n}\left[\sigma_{u}^{2}+\left(\frac{\mu_{Y}}{\mu_{X}}\right)^{2} \sigma_{v}^{2}\right]
\end{aligned}
$$

where $\mu_{X}$ denotes the population mean of $X$.

Existing Estimators: Manisha and Singh (2001) Estimator

Manisha and Singh (2001) proposed the estimator

$$
\bar{y}_{\theta}=\theta t_{R}+(1-\theta) \bar{y}
$$

where the bias of $\bar{y}_{\theta}$ is

$$
B\left(\bar{y}_{\theta}\right)=\theta\left\{\frac{\mu_{Y}}{n \mu_{X}^{2}}\left(\sigma_{X}^{2}+\sigma_{v}^{2}\right)-\frac{1}{n \mu_{X}} \rho \sigma_{X} \sigma_{Y}\right\}
$$

and the mean squared error is

$$
\begin{aligned}
& B\left(\bar{y}_{\theta}\right)= \\
& \frac{\sigma_{Y}^{2}}{n}\left[1-\theta \frac{C_{X}}{C_{Y}}\left(2 \rho-\theta \frac{C_{X}}{C_{Y}}\right)\right]+\frac{1}{n}\left[\theta^{2} \frac{\mu_{Y}^{2}}{\mu_{X}^{2}} \sigma_{V}^{2}+\sigma_{U}^{2}\right]
\end{aligned}
$$

where $\theta$ is a characterizing scalar and $U$ and $V$ are measurement errors corresponding to $Y$ and $X$ respectively.

Proposed Estimator(s)

The two parameter F-T estimators proposed are:

$$
\left.\begin{array}{l}
\bar{y}_{F T 1}^{\bullet}=\bar{y} T_{1} T_{2} \\
\bar{y}_{F T 2}^{\bullet}=\bar{y} T_{1} T_{2}^{-1} \\
\bar{y}_{F T 3}^{\bullet}=\bar{y} T_{1}^{-1} T_{2}
\end{array}\right\}
$$

where

$$
f=n / N
$$

and

$$
\left.\begin{array}{l}
\mathrm{T}_{\mathrm{i}}=\frac{\left(\mathrm{A}_{\mathrm{i}}+\mathrm{C}_{\mathrm{i}}\right) \overline{\mathrm{X}}_{\mathrm{i}}+\mathrm{fB}_{\mathrm{i}} \overline{\mathrm{x}}_{\mathrm{i}}}{\left(\mathrm{A}_{\mathrm{i}}+\mathrm{fB}_{\mathrm{i}}\right) \overline{\mathrm{X}}_{\mathrm{i}}+\mathrm{C}_{\mathrm{i}} \overline{\mathrm{x}}_{\mathrm{i}}} \\
\mathrm{A}=(\mathrm{K}-1)(\mathrm{K}-2) \\
\mathrm{B}=(\mathrm{K}-1)(\mathrm{K}-4) \\
\mathrm{C}=(\mathrm{K}-2)(\mathrm{K}-3)(\mathrm{K}-4)
\end{array}\right\}
$$

Thus,

$$
\begin{aligned}
& \bar{y}_{F T 1}^{\bullet}= \\
& \bar{y} \frac{\left(A_{1}+C_{1}\right) \bar{X}_{1}+f B_{1} \bar{x}_{1}}{\left(A_{1}+f B_{1}\right) \bar{X}_{1}+C_{1} \bar{x}_{1}} \frac{\left(A_{2}+C_{2}\right) \bar{X}_{2}+f B_{2} \bar{x}_{2}}{\left(A_{2}+f B_{2}\right) \bar{X}_{2}+C_{2} \bar{x}_{2}},
\end{aligned}
$$

$$
\begin{aligned}
& \bar{y}_{F T 2}^{\bullet}= \\
& \bar{y} \frac{\left(A_{1}+C_{1}\right) \bar{X}_{1}+f B_{1} \bar{x}_{1}}{\left(A_{1}+f B_{1}\right) \bar{X}_{1}+C_{1} \bar{x}_{1}} \frac{\left(A_{2}+f B_{2}\right) \bar{X}_{2}+C_{2} \bar{x}_{2}}{\left(A_{2}+C_{2}\right) \bar{X}_{2}+f B_{2} \bar{x}_{2}}
\end{aligned}
$$

and

$$
\begin{aligned}
& \bar{y}_{F T 3}^{\bullet}= \\
& \quad \bar{y} \frac{\left(A_{1}+f B_{1}\right) \bar{X}_{1}+C_{1} \bar{x}_{1}}{\left(A_{1}+C_{1}\right) \bar{X}_{1}+f B_{1} \bar{x}_{1}} \frac{\left(A_{2}+C_{2}\right) \bar{X}_{2}+f B_{2} \bar{x}_{2}}{\left(A_{2}+f B_{2}\right) \bar{X}_{2}+C_{2} \bar{x}_{2}} .
\end{aligned}
$$


Table 5.1: Members of the Proposed Class(es)

\begin{tabular}{|c|c|c|c|c|}
\hline \multirow{2}{*}{$K_{l}$} & \multicolumn{4}{|c|}{$K_{2}$} \\
\hline & 1 & 2 & 3 & 4 \\
\hline 1 & $t_{1}=\bar{y} \frac{\bar{X}_{1}}{\bar{x}_{1}} \frac{\bar{X}_{2}}{\bar{x}_{2}}$ & $t_{2}=\bar{y} \frac{\bar{X}_{1}}{\bar{x}_{1}} \frac{\bar{x}_{2}}{\bar{X}_{2}}$ & $t_{3}=\bar{y} \frac{\bar{X}_{1}}{\bar{x}_{1}} \frac{N \bar{X}_{2}-n \bar{x}_{2}}{(N-n) \bar{X}_{2}}$ & $t_{4}=\bar{y} \frac{\bar{X}_{1}}{\bar{x}_{1}}$ \\
\hline 2 & $t_{5}=\bar{y} \frac{\bar{x}_{1}}{\bar{X}_{1}} \frac{\bar{X}_{2}}{\bar{x}_{2}}$ & $t_{6}=\bar{y} \frac{\bar{x}_{1}}{\bar{X}_{1}} \frac{\bar{x}_{2}}{\bar{X}_{2}}$ & $t_{7}=\bar{y} \frac{\bar{x}_{1}}{\bar{X}_{1}} \frac{N \bar{X}_{2}-n \bar{x}_{2}}{(N-n) \bar{X}_{2}}$ & $t_{8}=\bar{y} \frac{\bar{x}_{1}}{\bar{X}_{1}}$ \\
\hline 3 & $t_{9}=\bar{y} \frac{N \bar{X}_{1}-n \bar{x}_{1}}{(N-n) \bar{X}_{1}} \frac{\bar{X}_{2}}{\bar{x}_{2}}$ & $t_{10}=\bar{y} \frac{N \bar{X}_{1}-n \bar{x}_{1}}{(N-n) \bar{X}_{1}} \frac{\bar{x}_{2}}{\bar{X}_{2}}$ & $t_{11}=\bar{y} \frac{N \bar{X}_{1}-n \bar{x}_{1}}{(N-n) \bar{X}_{1}} \frac{N \bar{X}_{2}-n \bar{x}_{2}}{(N-n) \bar{X}_{2}}$ & $t_{12}=\bar{y} \frac{N \bar{X}_{1}-n \bar{x}_{1}}{(N-n) \bar{X}_{1}}$ \\
\hline 4 & $t_{13}=\bar{y} \frac{\bar{X}_{2}}{\bar{x}_{2}}$ & $t_{14}=\bar{y} \frac{\bar{x}_{2}}{\bar{X}_{2}}$ & $t_{15}=\bar{y} \frac{N \bar{X}_{2}-n \bar{x}_{2}}{(N-n) \bar{X}_{2}}$ & $\bar{y}$ \\
\hline
\end{tabular}

Note that there is a combination of $K_{i}$ where $i=(1,2)$ where $\left(K_{1}=K_{2}\right)$ (see Table 5.1 for factors). When $K_{i}$ where $i=(1,2)$ is constant, it is important to choose suitably so that the resultant mean squared error of the proposed estimators may be minimized to the greatest extent. Using the proposed estimator many different estimators may be obtained because an estimator exists for each combination of $\left(K_{1}, K_{2}\right)$.

Properties of the Proposed Estimator(s)

For the approximation assume that:

$$
\begin{aligned}
& \delta_{0}=\frac{1}{\sqrt{n}}\left(W_{Y}+W_{U}\right) ; \\
& \delta_{1}=\frac{1}{\sqrt{n}}\left(W_{X_{1}}+W_{V}\right) ; \\
& \delta_{2}=\frac{1}{\sqrt{n}}\left(W_{X_{2}}+W_{T}\right) ;
\end{aligned}
$$

$$
\theta_{i}=\alpha_{i}-\beta_{i}
$$

$$
f=\frac{n}{N}
$$

$$
\alpha_{i}=\frac{f B_{i}}{A_{i}+f B_{i}+C_{i}}
$$

and

$$
\beta_{i}=\frac{C_{i}}{A_{i}+f B_{i}+C_{i}} .
$$

Theorem 6.1

The estimator $\bar{y}_{F T 1}^{\bullet}$ up to first order of approximation can be expressed as:

$$
\begin{aligned}
& \bar{y}_{F T 1}^{\bullet}=\bar{Y}+\delta_{0}+\frac{\bar{Y} \theta_{1}}{\bar{X}_{1}} \delta_{1}+\frac{\bar{Y} \theta_{2}}{\bar{X}_{2}} \delta_{2}-\frac{\bar{Y} \beta_{1} \theta_{1}}{\bar{X}_{1}^{2}} \delta_{1}^{2} \\
& -\frac{\bar{Y} \beta_{2} \theta_{2}}{\bar{X}_{2}^{2}} \delta_{2}^{2}+\frac{\theta_{1}}{\bar{X}_{1}} \delta_{0} \delta_{1}+\frac{\theta_{2}}{\bar{X}_{2}} \delta_{0} \delta_{2}+\frac{\bar{Y} \theta_{1} \theta_{2}}{\bar{X}_{1} \bar{X}_{2}} \delta_{1} \delta_{2}
\end{aligned}
$$

and the bias of $\bar{y}_{F T 1}^{\bullet}$ is: 


$$
\begin{aligned}
& \operatorname{Bias}\left(\overline{\mathrm{y}}_{\mathrm{FT} 1}^{\cdot}\right)=\frac{\overline{\mathrm{Y}}}{\mathrm{n}}\left\{\theta_{1} \rho_{01} \mathrm{C}_{0} \mathrm{C}_{1}+\theta_{2} \rho_{02} \mathrm{C}_{0} \mathrm{C}_{2}\right. \\
& \left.+\theta_{1} \theta_{2} \rho_{12} \mathrm{C}_{1} \mathrm{C}_{2}-\beta_{1} \theta_{1} \mathrm{C}_{1}^{2}\left(1+\frac{\sigma_{\mathrm{V}}^{2}}{\sigma_{\mathrm{X}_{1}}^{2}}\right)-\beta_{2} \theta_{2} \mathrm{C}_{2}^{2}\left(1+\frac{\sigma_{\mathrm{T}}^{2}}{\sigma_{\mathrm{X}_{2}}^{2}}\right)\right\}
\end{aligned}
$$

The mean squared error of $\bar{y}_{F T 1}^{\bullet}$ is:

$$
\begin{aligned}
& \operatorname{MSE}\left(\bar{y}_{F T 1}^{\bullet}\right)= \\
& \frac{1}{n}\left(\sigma_{Y}^{2}+\sigma_{U}^{2}\right)+\frac{\bar{Y}^{2}}{n}\left[\theta_{1}^{2} C_{1}^{2}\left(1+\frac{\sigma_{V}^{2}}{\sigma_{X_{1}}^{2}}\right)\right. \\
& +\theta_{2}^{2} C_{2}^{2}\left(1+\frac{\sigma_{T}^{2}}{\sigma_{X_{2}}^{2}}\right)+2 \theta_{1} \rho_{01} C_{0} C_{1} \\
& \left.+2 \theta_{2} \rho_{02} C_{0} C_{2}+2 \theta_{1} \theta_{2} \rho_{12} C_{1} C_{2}\right]
\end{aligned}
$$

Proof 6.1

From (5.2a) the proposed estimator is

$$
\begin{gathered}
\bar{y}_{F T 1}^{\bullet}= \\
\bar{y} \frac{\left(A_{1}+C_{1}\right) \bar{X}_{1}+f B_{1} \bar{x}_{1}}{\left(A_{1}+f B_{1}\right) \bar{X}_{1}+C_{1} \bar{x}_{1}} \frac{\left(A_{2}+C_{2}\right) \bar{X}_{2}+f B_{2} \bar{x}_{2}}{\left(A_{2}+f B_{2}\right) \bar{X}_{2}+C_{2} \bar{x}_{2}} \\
\bar{y}_{F T 1}^{\bullet}=\left[\bar{Y}+\delta_{0}\right]\left\{1+\frac{\alpha_{1} \delta_{1}}{\bar{X}_{1}}\right\}\left\{1+\frac{\alpha_{2} \delta_{2}}{\bar{X}_{2}}\right\} \\
\left\{1+\frac{\beta_{1} \delta_{1}}{\bar{X}_{1}}\right\}^{-1}\left\{1+\frac{\beta_{2} \delta_{2}}{\bar{X}_{2}}\right\}^{-1}
\end{gathered}
$$

and from this,

$$
\begin{aligned}
\bar{y}_{F T 1}^{\bullet}= & \bar{Y}+\delta_{0}+\frac{\bar{Y} \theta_{1}}{\bar{X}_{1}} \delta_{1}+\frac{\bar{Y} \theta_{2}}{\bar{X}_{2}} \delta_{2} \\
& -\frac{\bar{Y} \beta_{1} \theta_{1}}{\bar{X}_{1}^{2}} \delta_{1}^{2}-\frac{\bar{Y} \beta_{2} \theta_{2}}{\bar{X}_{2}^{2}} \delta_{2}^{2} \\
& +\frac{\theta_{1}}{\bar{X}_{1}} \delta_{0} \delta_{1}+\frac{\theta_{2}}{\bar{X}_{2}} \delta_{0} \delta_{2}+\frac{\bar{Y} \theta_{1} \theta_{2}}{\bar{X}_{1} \bar{X}_{2}} \delta_{1} \delta_{2}
\end{aligned}
$$$$
\text { and }
$$

$$
\begin{aligned}
\bar{y}_{F T 1}^{\bullet}-\bar{Y}= & \delta_{0}+\frac{\bar{Y} \theta_{1}}{\bar{X}_{1}} \delta_{1}+\frac{\bar{Y} \theta_{2}}{\bar{X}_{2}} \delta_{2} \\
& -\frac{\bar{Y} \beta_{1} \theta_{1}}{\bar{X}_{1}^{2}} \delta_{1}^{2}-\frac{\bar{Y} \beta_{2} \theta_{2}}{\bar{X}_{2}^{2}} \delta_{2}^{2} \\
& +\frac{\theta_{1}}{\bar{X}_{1}} \delta_{0} \delta_{1}+\frac{\theta_{2}}{\bar{X}_{2}} \delta_{0} \delta_{2}+\frac{\bar{Y} \theta_{1} \theta_{2}}{\bar{X}_{1} \bar{X}_{2}} \delta_{1} \delta_{2} .
\end{aligned}
$$

Thus, for the solution:

$$
\begin{aligned}
& {\left[\bar{y}_{F T 1}^{\bullet}-\bar{Y}\right]^{2}=\left\{\delta_{0}+\frac{\bar{Y} \theta_{1}}{\bar{X}_{1}} \delta_{1}+\frac{\bar{Y} \theta_{2}}{\bar{X}_{2}} \delta_{2}\right.} \\
& -\frac{\bar{Y} \beta_{1} \theta_{1}}{\bar{X}_{1}^{2}} \delta_{1}^{2}-\frac{\bar{Y} \beta_{2} \theta_{2}}{\bar{X}_{2}^{2}} \delta_{2}^{2}+\frac{\theta_{1}}{\bar{X}_{1}} \delta_{0} \delta_{1} \\
& \left.+\frac{\theta_{2}}{\bar{X}_{2}} \delta_{0} \delta_{2}+\frac{\bar{Y} \theta_{1} \theta_{2}}{\bar{X}_{1} \bar{X}_{2}} \delta_{1} \delta_{2}\right\}^{2}
\end{aligned}
$$

and

$$
\begin{aligned}
& \mathrm{E}\left[\overline{\mathrm{y}}_{\mathrm{FT} 1}^{\cdot}-\overline{\mathrm{Y}}\right]^{2}=\frac{1}{\mathrm{n}}\left(\sigma_{\mathrm{Y}}^{2}+\sigma_{\mathrm{U}}^{2}\right) \\
& +\frac{\overline{\mathrm{Y}}^{2}}{\mathrm{n}}\left[\theta_{1}^{2} \mathrm{C}_{1}^{2}\left(1+\frac{\sigma_{\mathrm{v}}^{2}}{\sigma_{\mathrm{x}_{1}}^{2}}\right)+\theta_{2}^{2} \mathrm{C}_{2}^{2}\left(1+\frac{\sigma_{\mathrm{T}}^{2}}{\sigma_{\mathrm{X}_{2}}^{2}}\right)\right. \\
& \left.+2 \theta_{1} \rho_{01} \mathrm{C}_{0} \mathrm{C}_{1}+2 \theta_{2} \rho_{02} \mathrm{C}_{0} \mathrm{C}_{2}+2 \theta_{1} \theta_{2} \rho_{12} \mathrm{C}_{1} \mathrm{C}_{2}\right] .
\end{aligned}
$$

Theorem 6.2

The estimator $\bar{y}_{F T 2}^{\bullet}$ up to first order of approximation can be expressed as:

$$
\begin{aligned}
& \overline{\mathrm{Y}}_{\mathrm{FT} 2}^{\cdot}=\overline{\mathrm{Y}}+\delta_{0}+\frac{\overline{\mathrm{Y}} \theta_{1}}{\overline{\mathrm{X}}_{1}} \delta_{1}-\frac{\overline{\mathrm{Y}} \theta_{2}}{\overline{\mathrm{X}}_{2}} \delta_{2}-\frac{\overline{\mathrm{Y}} \beta_{1} \theta_{1}}{\overline{\mathrm{X}}_{1}^{2}} \delta_{1}^{2} \\
& +\frac{\overline{\mathrm{Y}} \alpha_{2} \theta_{2}}{\overline{\mathrm{X}}_{2}^{2}} \delta_{2}^{2}+\frac{\theta_{1}}{\overline{\mathrm{X}}_{1}} \delta_{0} \delta_{1}-\frac{\theta_{2}}{\overline{\mathrm{X}}_{2}} \delta_{0} \delta_{2}-\frac{\overline{\mathrm{Y}} \theta_{1} \theta_{2}}{\overline{\mathrm{X}}_{1} \overline{\mathrm{X}}_{2}} \delta_{1} \delta_{2} .
\end{aligned}
$$

The bias of $\bar{y}_{F T 2}^{\bullet}$ is: 


$$
\begin{aligned}
& \operatorname{Bias}\left(\bar{y}_{F T 2}^{\bullet}\right)= \\
& \frac{\bar{Y}}{n}\left\{\theta_{1} \rho_{01} C_{0} C_{1}-\theta_{2} \rho_{02} C_{0} C_{2}-\theta_{1} \theta_{2} \rho_{12} C_{1} C_{2}\right. \\
& \left.\quad-\beta_{1} \theta_{1} C_{1}^{2}\left(1+\frac{\sigma_{V}^{2}}{\sigma_{X_{1}}^{2}}\right)+\alpha_{2} \theta_{2} C_{2}^{2}\left(1+\frac{\sigma_{T}^{2}}{\sigma_{X_{2}}^{2}}\right)\right\}
\end{aligned}
$$

and the mean squared error of $\bar{y}_{F T 2}^{\bullet}$ is:

$$
\begin{aligned}
& \operatorname{MSE}\left(\overline{\mathrm{y}}_{\mathrm{FT} 2}^{\cdot}\right)=\frac{1}{\mathrm{n}}\left(\sigma_{\mathrm{Y}}^{2}+\sigma_{\mathrm{v}}^{2}\right) \\
& +\frac{\overline{\mathrm{Y}}^{2}}{\mathrm{n}}\left[\theta_{1}^{2} \mathrm{C}_{1}^{2}\left(1+\frac{\sigma_{\mathrm{v}}^{2}}{\sigma_{\mathrm{x}_{1}}^{2}}\right)+\theta_{2}^{2} \mathrm{C}_{2}^{2}\left(1+\frac{\sigma_{\mathrm{T}}^{2}}{\sigma_{\mathrm{x}_{2}}^{2}}\right)\right. \\
& \left.+2 \theta_{1} \rho_{01} \mathrm{C}_{0} \mathrm{C}_{1}-2 \theta_{2} \rho_{02} \mathrm{C}_{0} \mathrm{C}_{2}-2 \theta_{1} \theta_{2} \rho_{12} \mathrm{C}_{1} \mathrm{C}_{2}\right] .
\end{aligned}
$$

Proof 6.2

From $(5.2 b)$ the proposed estimator is

$$
\begin{gathered}
\bar{y}_{F T 2}^{\bullet}= \\
\bar{y} \frac{\left(A_{1}+C_{1}\right) \bar{X}_{1}+f B_{1} \bar{x}_{1}}{\left(A_{1}+f B_{1}\right) \bar{X}_{1}+C_{1} \bar{x}_{1}} \frac{\left(A_{2}+f B_{2}\right) \bar{X}_{2}+C_{2} \bar{x}_{2}}{\left(A_{2}+C_{2}\right) \bar{X}_{2}+f B_{2} \bar{x}_{2}} \\
\bar{y}_{F T 2}^{\bullet}=\left[\bar{Y}+\delta_{0}\right]\left\{1+\frac{\alpha_{1} \delta_{1}}{\bar{X}_{1}}\right\}\left\{1+\frac{\beta_{2} \delta_{2}}{\bar{X}_{2}}\right\} \\
\left\{1+\frac{\alpha_{2} \delta_{2}}{\bar{X}_{2}}\right\}^{-1}\left\{1+\frac{\beta_{1} \delta_{1}}{\bar{X}_{1}}\right\}^{-1}
\end{gathered}
$$

Solving the equations results in:

$$
\begin{aligned}
\bar{y}_{F T 2}^{\bullet}= & \bar{Y}+\delta_{0}+\frac{\bar{Y} \theta_{1}}{\bar{X}_{1}} \delta_{1}-\frac{\bar{Y} \theta_{2}}{\bar{X}_{2}} \delta_{2} \\
& -\frac{\bar{Y} \beta_{1} \theta_{1}}{\bar{X}_{1}^{2}} \delta_{1}^{2}+\frac{\bar{Y} \alpha_{2} \theta_{2}}{\bar{X}_{2}^{2}} \delta_{2}^{2} \\
& +\frac{\theta_{1}}{\bar{X}_{1}} \delta_{0} \delta_{1} \frac{\theta_{2}}{\bar{X}_{2}} \delta_{0} \delta_{2}-\frac{\bar{Y} \theta_{1} \theta_{2}}{\bar{X}_{1} \bar{X}_{2}} \delta_{1} \delta_{2},
\end{aligned}
$$

$$
\begin{aligned}
& \bar{y}_{F T 2}^{\bullet}-\bar{Y}=\delta_{0}+\frac{\bar{Y} \theta_{1}}{\bar{X}_{1}} \delta_{1}-\frac{\bar{Y} \theta_{2}}{\bar{X}_{2}} \delta_{2} \\
& -\frac{\bar{Y} \beta_{1} \theta_{1}}{\bar{X}_{1}^{2}} \delta_{1}^{2}+\frac{\bar{Y} \alpha_{2} \theta_{2}}{\bar{X}_{2}^{2}} \delta_{2}^{2} \\
& +\frac{\theta_{1}}{\bar{X}_{1}} \delta_{0} \delta_{1}-\frac{\theta_{2}}{\bar{X}_{2}} \delta_{0} \delta_{2}-\frac{\bar{Y} \theta_{1} \theta_{2}}{\bar{X}_{1} \bar{X}_{2}} \delta_{1} \delta_{2}, \\
& \mathrm{E}\left[\overline{\mathrm{Y}}_{\mathrm{FT} 2}^{\bullet}-\overline{\mathrm{Y}}\right]=\mathrm{E}\left[\delta_{0}+\frac{\overline{\mathrm{Y}} \theta_{1}}{\overline{\mathrm{X}}_{1}} \delta_{1}-\frac{\overline{\mathrm{Y}} \theta_{2}}{\overline{\mathrm{X}}_{2}} \delta_{2}\right. \\
& -\frac{\overline{\mathrm{Y}} \beta_{1} \theta_{1}}{\overline{\mathrm{X}}_{1}^{2}} \delta_{1}^{2}+\frac{\overline{\mathrm{Y}} \alpha_{2} \theta_{2}}{\overline{\mathrm{X}}_{2}^{2}} \delta_{2}^{2}+\frac{\theta_{1}}{\overline{\mathrm{X}}_{1}} \delta_{0} \delta_{1} \\
& \left.-\frac{\theta_{2}}{\bar{X}_{2}} \delta_{0} \delta_{2}-\frac{\bar{Y} \theta_{1} \theta_{2}}{\bar{X}_{1} \bar{X}_{2}} \delta_{1} \delta_{2}\right] \\
& E\left[\bar{y}_{F T 2}^{\bullet}-\bar{Y}\right]=\frac{\bar{Y}}{n}\left\{\theta_{1} \rho_{01} C_{0} C_{1}-\theta_{2} \rho_{02} C_{0} C_{2}\right. \\
& -\theta_{1} \theta_{2} \rho_{12} C_{1} C_{2}-\beta_{1} \theta_{1} C_{1}^{2}\left(1+\frac{\sigma_{V}^{2}}{\sigma_{X_{1}}^{2}}\right) \\
& \left.+\alpha_{2} \theta_{2} C_{2}^{2}\left(1+\frac{\sigma_{T}^{2}}{\sigma_{X_{2}}^{2}}\right)\right\}
\end{aligned}
$$

and

$$
\begin{gathered}
\mathrm{E}\left[\overline{\mathrm{Y}}_{\mathrm{FT} 2}^{\cdot}-\overline{\mathrm{Y}}\right]^{2}=\mathrm{E}\left[\delta_{0}+\frac{\overline{\mathrm{Y}} \theta_{1}}{\overline{\mathrm{X}}_{1}} \delta_{1}-\frac{\overline{\mathrm{Y}} \theta_{2}}{\overline{\mathrm{X}}_{2}} \delta_{2}\right. \\
-\frac{\overline{\mathrm{Y}} \beta_{1} \theta_{1}}{\overline{\mathrm{X}}_{1}^{2}} \delta_{1}^{2}+\frac{\overline{\mathrm{Y}} \alpha_{2} \theta_{2}}{\overline{\mathrm{X}}_{2}^{2}} \delta_{2}^{2}+\frac{\theta_{1}}{\overline{\mathrm{X}}_{1}} \delta_{0} \delta_{1} \\
\left.-\frac{\theta_{2}}{\overline{\mathrm{X}}_{2}} \delta_{0} \delta_{2}-\frac{\overline{\mathrm{Y}} \theta_{1} \theta_{2}}{\overline{\mathrm{X}}_{1} \overline{\mathrm{X}}_{2}} \delta_{1} \delta_{2}\right]^{2}
\end{gathered}
$$

Based on the solution:

$$
\begin{aligned}
& \mathrm{E}\left[\overline{\mathrm{y}}_{\mathrm{FT} 2}^{\cdot}-\overline{\mathrm{Y}}\right]^{2}=\frac{1}{\mathrm{n}}\left(\sigma_{\mathrm{Y}}^{2}+\sigma_{\mathrm{U}}^{2}\right) \\
& +\frac{\overline{\mathrm{Y}}^{2}}{\mathrm{n}}\left[\theta_{1}^{2} \mathrm{C}_{1}^{2}\left(1+\frac{\sigma_{\mathrm{v}}^{2}}{\sigma_{\mathrm{x}_{1}}^{2}}\right)+\theta_{2}^{2} \mathrm{C}_{2}^{2}\left(1+\frac{\sigma_{\mathrm{T}}^{2}}{\sigma_{\mathrm{X}_{2}}^{2}}\right)\right. \\
& \left.+2 \theta_{1} \rho_{01} \mathrm{C}_{0} \mathrm{C}_{1}-2 \theta_{2} \rho_{02} \mathrm{C}_{0} \mathrm{C}_{2}-2 \theta_{1} \theta_{2} \rho_{12} \mathrm{C}_{1} \mathrm{C}_{2}\right] .
\end{aligned}
$$


Theorem 6.3

The estimator $\bar{y}_{F T 3}^{\bullet}$, up to first order of approximation, can be expressed as:

$$
\begin{aligned}
\bar{y}_{F T 3}^{\bullet}= & \bar{Y}+\delta_{0}-\frac{\bar{Y} \theta_{1}}{\bar{X}_{1}} \delta_{1}+\frac{\bar{Y} \theta_{2}}{\bar{X}_{2}} \delta_{2} \\
& +\frac{\bar{Y} \alpha_{1} \theta_{1}}{\bar{X}_{1}^{2}} \delta_{1}^{2}-\frac{\bar{Y} \beta_{2} \theta_{2}}{\bar{X}_{2}^{2}} \delta_{2}^{2} \\
& -\frac{\theta_{1}}{\bar{X}_{1}} \delta_{0} \delta_{1}+\frac{\theta_{2}}{\bar{X}_{2}} \delta_{0} \delta_{2}-\frac{\bar{Y} \theta_{1} \theta_{2}}{\bar{X}_{1} \bar{X}_{2}} \delta_{1} \delta_{2}
\end{aligned}
$$

and the bias of $\bar{y}_{F T 3}^{\bullet}$ is:

$$
\begin{aligned}
& \operatorname{Bias}\left(\overline{\mathrm{y}}_{\mathrm{FT} 3}^{\cdot}\right)=\frac{\overline{\mathrm{Y}}}{\mathrm{n}}\left\{\theta_{2} \rho_{02} \mathrm{C}_{0} \mathrm{C}_{2}-\theta_{1} \rho_{01} \mathrm{C}_{0} \mathrm{C}_{1}\right. \\
& -\theta_{1} \theta_{2} \rho_{12} \mathrm{C}_{1} \mathrm{C}_{2}+\alpha_{1} \theta_{1} \mathrm{C}_{1}^{2}\left(1+\frac{\sigma_{\mathrm{V}}^{2}}{\sigma_{\mathrm{X}_{1}}^{2}}\right) \\
& \left.-\beta_{2} \theta_{2} \mathrm{C}_{2}^{2}\left(1+\frac{\sigma_{\mathrm{T}}^{2}}{\sigma_{\mathrm{X}_{2}}^{2}}\right)\right\}
\end{aligned}
$$

The mean squared error of $\bar{y}_{F T 3}^{\bullet}$ is:

$$
\begin{aligned}
& \operatorname{MSE}\left(\overline{\mathrm{y}}_{\mathrm{FT} 3}^{\cdot}\right)=\frac{1}{\mathrm{n}}\left(\sigma_{\mathrm{Y}}^{2}+\sigma_{\mathrm{v}}^{2}\right) \\
& +\frac{\overline{\mathrm{Y}}^{2}}{\mathrm{n}}\left[\theta_{1}^{2} \mathrm{C}_{1}^{2}\left(1+\frac{\sigma_{\mathrm{v}}^{2}}{\sigma_{\mathrm{x}_{1}}^{2}}\right)+\theta_{2}^{2} \mathrm{C}_{2}^{2}\left(1+\frac{\sigma_{\mathrm{T}}^{2}}{\sigma_{\mathrm{x}_{2}}^{2}}\right)\right. \\
& \left.-2 \theta_{1} \rho_{01} \mathrm{C}_{0} \mathrm{C}_{1}+2 \theta_{2} \rho_{02} \mathrm{C}_{0} \mathrm{C}_{2}-2 \theta_{1} \theta_{2} \rho_{12} \mathrm{C}_{1} \mathrm{C}_{2}\right] .
\end{aligned}
$$

Proof 6.3

From (5.2c) the proposed estimator is

$$
\begin{aligned}
& \bar{y}_{F T 3}^{\bullet}= \\
& \quad \bar{y} \frac{\left(A_{1}+f B_{1}\right) \bar{X}_{1}+C_{1} \bar{x}_{1}}{\left(A_{1}+C_{1}\right) \bar{X}_{1}+f B_{1} \bar{x}_{1}} \frac{\left(A_{2}+C_{2}\right) \bar{X}_{2}+f B_{2} \bar{x}_{2}}{\left(A_{2}+f B_{2}\right) \bar{X}_{2}+C_{2} \bar{x}_{2}}
\end{aligned}
$$

$$
\begin{aligned}
\overline{\mathrm{y}}_{\mathrm{FT} 3}^{\bullet}= & {\left[\overline{\mathrm{Y}}+\delta_{0}\right]\left\{1+\frac{\alpha_{2} \delta_{2}}{\overline{\mathrm{X}}_{2}}\right\}\left\{1+\frac{\beta_{1} \delta_{1}}{\overline{\mathrm{X}}_{1}}\right\} } \\
& \left\{1+\frac{\alpha_{1} \delta_{1}}{\overline{\mathrm{X}}_{1}}\right\}^{-1}\left\{1+\frac{\beta_{2} \delta_{2}}{\overline{\mathrm{X}}_{2}}\right\}^{-1},
\end{aligned}
$$

which results in

$$
\begin{aligned}
\bar{y}_{F T 3}^{\bullet}= & \bar{Y}+\delta_{0}-\frac{\bar{Y} \theta_{1}}{\bar{X}_{1}} \delta_{1}+\frac{\bar{Y} \theta_{2}}{\bar{X}_{2}} \delta_{2} \\
+ & \frac{\bar{Y} \alpha_{1} \theta_{1}}{\bar{X}_{1}^{2}} \delta_{1}^{2}-\frac{\bar{Y} \beta_{2} \theta_{2}}{\bar{X}_{2}^{2}} \delta_{2}^{2} \\
& -\frac{\theta_{1}}{\bar{X}_{1}} \delta_{0} \delta_{1}+\frac{\theta_{2}}{\bar{X}_{2}} \delta_{0} \delta_{2}-\frac{\bar{Y} \theta_{1} \theta_{2}}{\bar{X}_{1} \bar{X}_{2}} \delta_{1} \delta_{2}, \\
\bar{y}_{F T 3}^{\bullet}-\bar{Y}= & \delta_{0}-\frac{\bar{Y} \theta_{1}}{\bar{X}_{1}} \delta_{1}+\frac{\bar{Y} \theta_{2}}{\bar{X}_{2}} \delta_{2} \\
& +\frac{\bar{Y}_{1} \theta_{1}}{\bar{X}_{1}^{2}} \delta_{1}^{2}-\frac{\bar{Y} \beta_{2} \theta_{2}}{\bar{X}_{2}^{2}} \delta_{2}^{2} \\
& -\frac{\theta_{1}}{\bar{X}_{1}} \delta_{0} \delta_{1}+\frac{\theta_{2}}{\bar{X}_{2}} \delta_{0} \delta_{2}-\frac{\bar{Y}_{1} \theta_{2}}{\bar{X}_{1} \bar{X}_{2}} \delta_{1} \delta_{2},
\end{aligned}
$$$$
\begin{aligned}
& E\left[\bar{y}_{F T 3}^{\bullet}-\bar{Y}\right]= \\
& E\left[\begin{array}{l}
\delta_{0}-\frac{\bar{Y} \theta_{1}}{\bar{X}_{1}} \delta_{1}+\frac{\bar{Y} \theta_{2}}{\bar{X}_{2}} \delta_{2} \\
+\frac{\bar{Y} \alpha_{1} \theta_{1}}{\bar{X}_{1}^{2}} \delta_{1}^{2}-\frac{\bar{Y} \beta_{2} \theta_{2}}{\bar{X}_{2}^{2}} \delta_{2}^{2} \\
-\frac{\theta_{1}}{\bar{X}_{1}} \delta_{0} \delta_{1}+\frac{\theta_{2}}{\bar{X}_{2}} \delta_{0} \delta_{2}-\frac{\bar{Y} \theta_{1} \theta_{2}}{\bar{X}_{1} \bar{X}_{2}} \delta_{1} \delta_{2}
\end{array}\right]
\end{aligned}
$$

and 


$$
\begin{gathered}
\mathrm{E}\left[\overline{\mathrm{y}}_{\mathrm{FT} 3}^{\bullet}-\overline{\mathrm{Y}}\right]=\frac{\overline{\mathrm{Y}}}{\mathrm{n}}\left\{\theta_{2} \rho_{02} \mathrm{C}_{0} \mathrm{C}_{2}-\theta_{1} \rho_{01} \mathrm{C}_{0} \mathrm{C}_{1}\right. \\
-\theta_{1} \theta_{2} \rho_{12} \mathrm{C}_{1} \mathrm{C}_{2}+\alpha_{1} \theta_{1} \mathrm{C}_{1}^{2}\left(1+\frac{\sigma_{\mathrm{V}}^{2}}{\sigma_{\mathrm{X}_{1}}^{2}}\right) \\
\left.-\beta_{2} \theta_{2} \mathrm{C}_{2}^{2}\left(1+\frac{\sigma_{\mathrm{T}}^{2}}{\sigma_{\mathrm{X}_{2}}^{2}}\right)\right\} .
\end{gathered}
$$

From which follows

$$
\begin{aligned}
& {\left[\bar{y}_{F T 3}^{\bullet}-\bar{Y}\right]^{2}=\left[\delta_{0}-\frac{\bar{Y} \theta_{1}}{\bar{X}_{1}} \delta_{1}+\frac{\bar{Y} \theta_{2}}{\bar{X}_{2}} \delta_{2}+\frac{\bar{Y} \alpha_{1} \theta_{1}}{\bar{X}_{1}^{2}} \delta_{1}^{2}\right.} \\
& \left.-\frac{\bar{Y} \beta_{2} \theta_{2}}{\bar{X}_{2}^{2}} \delta_{2}^{2}-\frac{\theta_{1}}{\bar{X}_{1}} \delta_{0} \delta_{1}+\frac{\theta_{2}}{\bar{X}_{2}} \delta_{0} \delta_{2}-\frac{\bar{Y} \theta_{1} \theta_{2}}{\bar{X}_{1} \bar{X}_{2}} \delta_{1} \delta_{2}\right]^{2}
\end{aligned}
$$

and

$$
\begin{gathered}
\mathrm{E}\left[\overline{\mathrm{Y}}_{\mathrm{FT} 3}^{\bullet}-\overline{\mathrm{Y}}\right]^{2}=\mathrm{E}\left[\delta_{0}-\frac{\overline{\mathrm{Y}} \theta_{1}}{\overline{\mathrm{X}}_{1}} \delta_{1}+\frac{\overline{\mathrm{Y}} \theta_{2}}{\overline{\mathrm{X}}_{2}} \delta_{2}\right. \\
+\frac{\overline{\mathrm{Y}} \alpha_{1} \theta_{1}}{\overline{\mathrm{X}}_{1}^{2}} \delta_{1}^{2}-\frac{\overline{\mathrm{Y}} \beta_{2} \theta_{2}}{\overline{\mathrm{X}}_{2}^{2}} \delta_{2}^{2}-\frac{\theta_{1}}{\overline{\mathrm{X}}_{1}} \delta_{0} \delta_{1} \\
\left.+\frac{\theta_{2}}{\overline{\mathrm{X}}_{2}} \delta_{0} \delta_{2}-\frac{\overline{\mathrm{Y}}_{1} \theta_{2}}{\overline{\mathrm{X}}_{1} \overline{\mathrm{X}}_{2}} \delta_{1} \delta_{2}\right]^{2}
\end{gathered}
$$

The solution of which results in:

$$
\begin{aligned}
& \mathrm{E}\left[\overline{\mathrm{y}}_{\mathrm{FT} 3}^{\circ}-\overline{\mathrm{Y}}\right]^{2}=\frac{1}{\mathrm{n}}\left(\sigma_{\mathrm{Y}}^{2}+\sigma_{\mathrm{U}}^{2}\right) \\
& +\frac{\overline{\mathrm{Y}}^{2}}{\mathrm{n}}\left[\theta_{1}^{2} \mathrm{C}_{1}^{2}\left(1+\frac{\sigma_{\mathrm{v}}^{2}}{\sigma_{\mathrm{x}_{1}}^{2}}\right)+\theta_{2}^{2} \mathrm{C}_{2}^{2}\left(1+\frac{\sigma_{\mathrm{T}}^{2}}{\sigma_{\mathrm{X}_{2}}^{2}}\right)\right. \\
& \left.-2 \theta_{1} \rho_{01} \mathrm{C}_{0} \mathrm{C}_{1}+2 \theta_{2} \rho_{02} \mathrm{C}_{0} \mathrm{C}_{2}-2 \theta_{1} \theta_{2} \rho_{12} \mathrm{C}_{1} \mathrm{C}_{2}\right] .
\end{aligned}
$$

Minimum Mean Squared Error \& Optimal Choices for the Proposed Estimator(s)

The mean squared error of the proposed estimators $\bar{y}_{F T 1}^{\bullet}, \quad \bar{y}_{F T 2}^{\bullet}$ and $\bar{y}_{F T 3}^{\bullet}$ shown in (6.3), (6.6) and (6.9) respectively, are functions with unknown parameter $\theta_{i} ; i=(1,2)$, whereas $\theta_{i}$ is a function of $K$ solely. Thus, it is practical to calculate an optimum value of $K$ in such a way that the mean squared error of the resultant proposed estimator becomes least.

Consider $\bar{y}_{F T 1}^{\bullet}$, notice the minimum mean squared error. On differentiation of $\operatorname{MSE}\left(\bar{y}_{F T 1}^{\bullet}\right)$ with respect to $\theta_{1}$ and $\theta_{2}$ and equating to zero (assuming $\theta_{i} \neq 0$ ), two simultaneous equations result:

$$
\begin{gathered}
C_{1}^{2}\left(1+\frac{\sigma_{V}^{2}}{\sigma_{X_{1}}^{2}}\right) \theta_{1}+\rho_{12} C_{1} C_{2} \theta_{2}+\rho_{01} C_{0} C_{1}=0 \\
\text { for } \frac{\partial}{\partial \theta_{1}}\left[E\left[\bar{y}_{F T 1}^{\bullet}-\bar{Y}\right]^{2}\right]=0
\end{gathered}
$$

and

$$
\begin{gathered}
C_{2}^{2}\left(1+\frac{\sigma_{T}^{2}}{\sigma_{X_{2}}^{2}}\right) \theta_{2}+\rho_{12} C_{1} C_{2} \theta_{1}+\rho_{02} C_{0} C_{2}=0 \\
\text { For } \frac{\partial}{\partial \theta_{2}}\left[E\left[\bar{y}_{F T 1}^{\bullet}-\bar{Y}\right]^{2}\right]=0 .
\end{gathered}
$$

From (7.1) and (7.2) the values of $\theta_{1}$ and $\theta_{2}$ are:

$$
\begin{aligned}
& \hat{\theta}_{(1)}=\frac{C_{0}}{C_{1}} \frac{\rho_{02} \rho_{12}-\rho_{01}\left(1+\frac{\sigma_{T}^{2}}{\sigma_{\mathrm{x}_{2}}^{2}}\right)}{\left[\left(1+\frac{\sigma_{\mathrm{v}}^{2}}{\sigma_{\mathrm{x}_{1}}^{2}}\right)\left(1+\frac{\sigma_{\mathrm{T}}^{2}}{\sigma_{\mathrm{x}_{2}}^{2}}\right)-\rho_{12}^{2}\right]} \\
& \left.\hat{\theta}_{(2)}=\frac{C_{0}}{C_{2}} \frac{\rho_{01} \rho_{12}-\rho_{02}\left(1+\frac{\sigma_{v}^{2}}{\sigma_{x_{1}}^{2}}\right)}{\left[\left(1+\frac{\sigma_{v}^{2}}{\sigma_{x_{1}}^{2}}\right)\left(1+\frac{\sigma_{T}^{2}}{\sigma_{x_{2}}^{2}}\right)-\rho_{12}^{2}\right]}\right\} \text {. }
\end{aligned}
$$

The optimum values $\hat{\theta}_{(1)}=\Delta_{1}$ and $\hat{\theta}_{(2)}=\Delta_{2}$, for example, provide a minimum 
mean squared error to $\bar{y}_{F T 1}^{\bullet}$, where the second derivative is positive. Similarly, $\hat{\theta}_{(3)}=\hat{\theta}_{(1)}$; $\hat{\theta}_{(4)}=(-1) \hat{\theta}_{(2)} \quad$ and $\quad \hat{\theta}_{(5)}=(-1) \hat{\theta}_{(1)}$; $\hat{\theta}_{(6)}=\hat{\theta}_{(2)}$ are optimal choices corresponding to $\bar{y}_{F T 2}^{\bullet}$ and $\bar{y}_{F T 3}^{\bullet}$ respectively. These $\theta_{(\bullet)}$ provide polynomials in terms of $K$ to produce values for which the mean squared error will be optimum.

Empirical Study

This illustration demonstrates how to evaluate the gain in efficiencies (in terms of mean squared error) obtained by the proposed estimators. To evaluate the performance of the various estimators discussed, a population is considered (see Appendix A); required information is shown in Table 8.1.

Table 8.1: Population Parameters

\begin{tabular}{|c|c|c|c|}
\hline Parameter & Value & Parameter & Value \\
\hline $\bar{Y}$ & 63.396 & $n$ & 50 \\
\hline $\bar{X}_{1}$ & 48.136 & $N$ & 250 \\
\hline $\bar{X}_{2}$ & 56.364 & $f$ & 0.2 \\
\hline$C_{0}$ & 0.2899 & $\rho_{01}$ & 0.8544 \\
\hline$C_{1}$ & 0.4637 & $\rho_{02}$ & 0.8249 \\
\hline$C_{2}$ & 0.4085 & $\rho_{12}$ & 0.8289 \\
\hline
\end{tabular}

Table 8.2: Percent Relative Efficiency of Various Estimators with respect to Mean per Unit Estimator

\begin{tabular}{|c|c|c|c|}
\hline \multirow{2}{*}{ Estimator(s) } & \multicolumn{3}{|c|}{$\begin{array}{l}P R E(\bullet) \text { with respect to MPU } \\
\text { Estimator }\end{array}$} \\
\hline & $\operatorname{MSE}\left(\bar{y}_{F T 1}^{\bullet}\right)$ & $\operatorname{MSE}\left(\bar{y}_{F T 2}^{\bullet}\right)$ & $\operatorname{MSE}\left(\bar{y}_{F T 3}^{\bullet}\right)$ \\
\hline $\bar{y}$ & 100 & 100 & 100 \\
\hline$t_{1}$ & 40.75 & 27.41 & 42.31 \\
\hline$t_{2}$ & 27.41 & 40.75 & 23.63 \\
\hline$t_{3}$ & 93.26 & 64.96 & 109.88 \\
\hline$t_{4}$ & 36.30 & 36.29 & 21.95 \\
\hline$t_{5}$ & 21.35 & 11.52 & 18.71 \\
\hline$t_{6}$ & 11.53 & 21.35 & 22.65 \\
\hline$t_{7}$ & 23.87 & 19.27 & 33.28 \\
\hline$t_{8}$ & 21.95 & 21.95 & 36.29 \\
\hline$t_{9}$ & 38.35 & 28.38 & 40.94 \\
\hline$t_{10}$ & 28.38 & 38.35 & 21.79 \\
\hline$t_{11}$ & 104.25 & 84.15 & 79.65 \\
\hline$t_{12}$ & 106.28 & 106.2 & 71.89 \\
\hline$t_{13}$ & 42.40 & 25.70 & 42.39 \\
\hline$t_{14}$ & 25.71 & 42.39 & 25.70 \\
\hline$t_{15}$ & 106.23 & 75.51 & 106.21 \\
\hline $\begin{array}{c}\text { Opt } \\
\left(\bar{y}_{F T 1}^{\bullet}\right)^{*}\end{array}$ & 113.05 & 92.27 & 95.01 \\
\hline $\begin{array}{c}\text { Opt } \\
\left(\bar{y}_{F T 2}^{\bullet}\right)^{*}\end{array}$ & 92.28 & 113.04 & 74.28 \\
\hline $\begin{array}{c}\text { Opt } \\
\left(\bar{y}_{F T 3}^{\bullet}\right)^{*}\end{array}$ & 95.02 & 74.28 & 113.04 \\
\hline
\end{tabular}


Results

Three different approaches were examined as tools for estimating in the presence of measurement error. Results indicate that the proposed approaches are effective and efficient over many existing strategies. The multiple choices for $K$ are accessible via:

$$
\begin{gathered}
\left(\Delta_{1}+1\right) K_{1}^{3}+\left(f \Delta_{1}-f-8 \Delta_{1}-9\right) K_{1}^{2} \\
+\left(23 \Delta_{1}-5 f \Delta_{1}+5 f+26\right) K_{1} \\
+\left(4 f \Delta_{1}-22 \Delta_{1}-4 f-24\right)=0
\end{gathered}
$$

and

$$
\begin{gathered}
\left(\Delta_{2}+1\right) K_{2}^{3}+\left(f \Delta_{2}-f-8 \Delta_{2}-9\right) K_{2}^{2} \\
\quad+\left(23 \Delta_{2}-5 f \Delta_{2}+5 f+26\right) K_{2} \\
+\left(4 f \Delta_{2}-22 \Delta_{2}-4 f-24\right)=0
\end{gathered}
$$

Polynomials (9.1) and (9.2), which are obtained from (7.3), provide three roots for lesser mean squared error. As discussed for $K_{1}=K_{2}=4$ the proposed classes provide mean per unit estimators, thus those values are unbiased estimators.

For the estimator $\bar{y}_{F T 1}^{\bullet}$, the optimum values of the characterizing scalar are $\left(K_{1}\right)_{1}=4.4951, \quad\left(K_{1}\right)_{2}=3.1167$, $\left(K_{1}\right)_{3}=1.8111, \quad\left(K_{2}\right)_{1}=4.5063$, $\left(K_{2}\right)_{2}=3.1133$ and $\left(K_{2}\right)_{3}=1.8096$. For $\bar{y}_{F T 2}^{\bullet}$, the values are $\left(K_{1}\right)_{4}=\left(K_{1}\right)_{1}$, $\left(K_{1}\right)_{5}=\left(K_{1}\right)_{2}, \quad\left(K_{1}\right)_{6}=\left(K_{1}\right)_{3} \quad$ and $\left(K_{2}\right)_{4}=1.8857$, and for the $\bar{y}_{F T 3}^{\bullet}$ estimator values are, $\left(K_{1}\right)_{7}=11.5039,\left(K_{2}\right)_{7}=\left(K_{2}\right)_{1}$, $\left(K_{2}\right)_{8}=\left(K_{2}\right)_{2}$ and $\left(K_{2}\right)_{9}=\left(K_{2}\right)_{3}$ with the remaining imaginary roots.

Tables 8.2 and 8.3 show that the proposed estimator is efficient over many currently used estimators, including the Manisha and Singh (2001) and the Shalabh (1997).

\section{Conclusion}

Based on study results, the proposed estimator(s) have several benefits over estimators currently used in research, including:

1. For different values of the characterizing scalar, there now exists a new estimation tool; and

2. The proposed class(es) provides a wide range for selecting the constant scalar by solving the associated polynomials and for root values estimators attains minimum mean squared error.

The proposed methodology is more effective, practicable and efficient, and may be recommended for use in practice.

\section{References}

Cheng, C., \& Van Ness, J. W. (1999). Statistical regression with measurement error. London, UK: Arnold Publishers.

Cochran, W. G. (2005). Sampling techniques. $2^{\text {nd }} E d$. New Delhi, India: Wiley Eastern Private Limited.

Dalabehara, M., \& Sahoo, L. N. (2000). An unbiased estimator in two-phase sampling using two auxiliary variables. Journal of Indian Society of Agricultural Statistics, 53(2), 134140.

Fuller, W. A. (1987). Measurement error models. New York, NY: Wiley.

Kadilar, C., \& Cingi, H. (2005). A new estimator using two auxiliary variables. Applied Mathematics and Computation, 162, 901-908.

Manisha, \& Singh, R. K. (2001). Estimation of population mean in the presence of measurement errors. Journal of Indian Society of Agricultural Statistics, 54(1), 13-18.

Mukhopadhyay, P. (2000). Theory and methods of survey sampling. New Delhi, India: Prentice Hall of India Pvt. Ltd.

Murthy, M. N. (1976). Sampling theory and methods. Calcutta, IN: Statistical Publishing Society.

Neter, J. (1970). Measurement errors in reports of consumer expenditures. Journal of Marketing Research, 7, 11-25. 


\section{DIWAKAR SHUKLA, SHARAD PATHAK \& NARENDRA SINGH THAKUR}

Shalabh. (1997). Ratio method of estimation in presence of measurement errors. Journal of Indian Society of Agricultural Statistics, 50(2), 150-155.

Shukla, D. (2002). F-T estimator under two-phase sampling. Metron, 59(1-2), 253-263.

Shukla, D., Thakur, N. S., Pathak, S., \& Rajput, D. S. (2009). Estimation of mean under imputation of missing data using factor-type estimator in two-phase sampling. Statistics in Transition, 10(3), 397-414.

Shukla, D., Thakur, N. S. Thakur, D. S., \& Pathak, S. (2011). Linear Combination Based Imputation Method for Missing Data in Sample. International Journal of Modern Engineering Research, 1(2), 580-596.

Shukla, D., Pathak, S., \& Thakur, N. S. (2012). Estimation of population mean using two auxiliary sources in sample surveys. Statistics in Transition, 13(1), 21-36.

Shukla, D., Pathak, S. , \& Thakur, N. S. (2012). An estimator for mean estimation in presence measurement error. Research \& Reviews: A Journal of Statistics, 1(1), 1-8.

Shukla, D., Pathak, S., \& Thakur, N. S. (2012). A transformed estimator for estimation of population mean with missing data in samplesurveys. Journal of Current Engineering Research, 2(1), 50-55.
Singh, H. P., \& Karpe, N. (2008). RatioProduct estimator for population mean in presence of measurement errors. Journal of Applied Statistical Sciences, 16(4), 49-64.

Singh, V. K., \& Shukla, D. (1987): One parameter family of factor-type ratio estimator. Metron, 45(1-2), 273-283.

Sud, U. C., Shrivastava, A. K., \& Sharma, D. P. (2001). On a biased estimator in repeat surveys. Journal of the Indian Society of Agricultural Statistics, 54(1), 29-42.

Sud, V. C., \& Shrivastava, A. K. (2000). Estimation and population mean in repeat surveys on the presence of measurement errors. Journal of Indian Society of Agricultural Statistics, 53(2), 125-133.

Sukhatme, P. V., Sukhatme, B. V., Sukhatme, S., \& Ashok, C. (1984). Sampling theory of surveys with applications. New Delhi: Iowa State University Press.

Thakur, N. S., Yadav, K., \& Pathak, S. (2011). Estimation of mean in presence of missing data under two-phase sampling scheme. Journal of Reliability and Statistical Studies, 4(2), 93-104.

Thakur, N. S., Yadav, K., \& Pathak, S. (2012). Some imputation methods in double sampling scheme for estimation of population mean. International Journal of Modern Engineering Research, 2(1), 200-207. 